\title{
Field Evaporation Behavior of Ternary Compound Semiconductor $\operatorname{In}_{\mathbf{x}} \mathrm{Al}_{1-\mathrm{x}} \mathbf{N}$
}

\author{
Baishakhi Mazumder ${ }^{1}$, Scott Broderick ${ }^{1}$, Krishna Rajan ${ }^{1}$ Joaquin Peralta $^{2}$, Humberto Foronda ${ }^{3}$ and \\ James S. Speck ${ }^{3}$ \\ 1. Department of Materials Design and Innovation, University at Buffalo, Buffalo, NY, USA \\ 2. Department Ciencias Fisicas, Universidad Andres Bello, Santiago, Chile \\ 3. Materials Department, University of California, Santa Barbara, CA, USA
}

III-nitride binary and ternary compound semiconductors are essential for the development of highfrequency and high-power electronic and optoelectronic devices operating in the ultraviolet spectral region due to their wide band gap and unique electronic characteristics. InAlN is attracting much attention due to its lattice matching capability to GaN substrates and its variable band gap energy which can be tuned from 1.9 to $6.2 \mathrm{eV}$ [1]. In order to facilitate high performance devices, structural and chemical information must be obtained and optimized at the nanoscale level. In the past decade, atom probe tomography (APT) has emerged as one of the most powerful techniques to analyze semiconductors and device structures at the sub-nanometer scale [2]. However, studying III-Nitride heterosturctures using atom probe has been a challenge due to compound semiconductors exhibiting a complex field evaporation behavior [3]. In this work we have made both experimental and theoretical efforts to understand the field evaporation behavior of ternary alloy $\operatorname{In}_{\mathrm{x}} \mathrm{Al}_{1-\mathrm{x}} \mathrm{N}$ in a multilayer stack with $\mathrm{GaN}$.

GaN/InAlN( 35 nm)/GaN multilayer structure shown in Figure (1a) was investigated in LEAP 4000X HR using UV laser pulses with pulse energy varying from 0.3-0.5 pJ. The base temperature of the tip was maintained at $30 \mathrm{~K}$. During the evaporation of this multilayered stack, a large voltage difference between the different layers was observed (Figure 1b), clearly demonstrating a significant field difference between the layers. The evaporation field difference between these dissimilar materials induces significant variations of the specimen surface curvature; hence there is ion projection trajectory that affects the 3D reconstruction shown in Figure (1c). The voltage ratio between $\mathrm{GaN}$ and InGaN are roughly estimated from the voltage plot as 1:1.4. As the evaporation field is calculated using $F=V / k R$, where $\mathrm{R}$ is the radius of the curvature and $\mathrm{k}$ is the geometrical factor, we can estimate the field ratio of GaN/InAlN equal to $1: 1.4$. The tip curvature ratio for high-on-low field (InAlN/GaN) material is higher than the low-on-high field (GaN/InAlN) material when approaching the interface. Indium composition plays a vital role in the field evaporation of this ternary alloy so different In concentrations were used to study the variation in the evaporation field.

To further assess the difference in evaporation fields of the layers, a first principles approach is utilized to calculate the evaporation field of $\mathrm{N}$ from both a GaN and InAlN surface, thereby defining the relative difference in electric fields. These calculations, as schematically shown in Figure 2, operate by calculating the field required to pull an atom from the surface of the material, and by tracking the change in field with distance from the surface, the evaporation field is extracted. We have utilized this approach previously for metallic systems [4,5], which we will expand here to a semiconducting multilayer system. Additionally, we will compare ion evaporation maps of the different layers to assess the evaporation physics. These maps compare the number and chemistry of multi-ion evaporations, which we can then assess versus the overall layer composition to understand the propensity of an atom to evaporate given the neighborhood chemistry / structure. This work will provide a computational protocol for identifying 
relative evaporation fields of layers, thereby providing a more reliable output and analysis of layered semi-conductor materials.

References:

[1] I. Vurgaftman, L.R. Ram-Mohan and J.R. Meyer, J. Appl. Phys. 89 (2001) p.5815.

[2] T. F Kelly and D.J. Larson, Annual Review of Materials Research 42 (2012) p.1.

[3] L. Mancini, The Journal of Physical Chemistry C, 118 (2014) p.24136.

[4] J. Peralta, S.R. Broderick, K. Rajan. Ultramicroscopy 132 (2013) p. 143.

[5] C. Loyola, J. Peralta, S.R. Broderick, K. Rajan, J. Vacuum Sci. Tech. A 34 (2016) p. 061404.

(a)

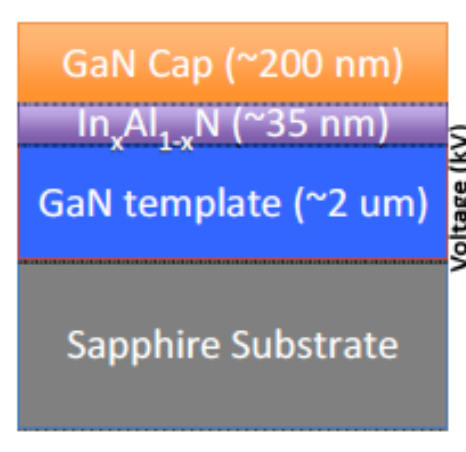

(b)

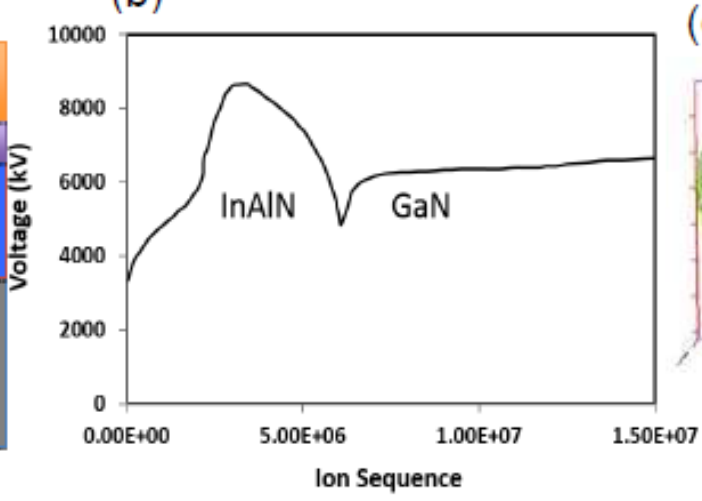

(c)

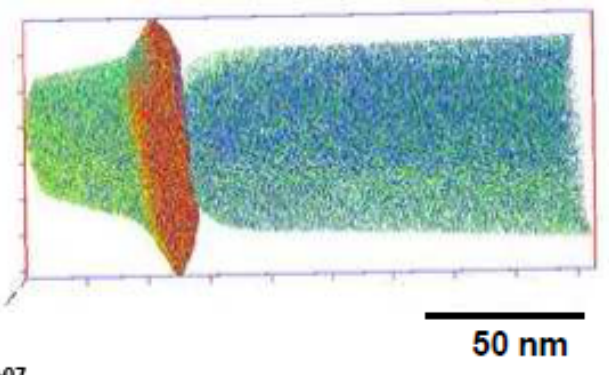

Figure 1. (a) Sample schematic (b) Voltage plot during field evaporation of GaN/InAlN/GaN showing the field difference between InAlN and GaN (c) 3D elemental map of the analyzed multilayer tip using voltage reconstruction method.
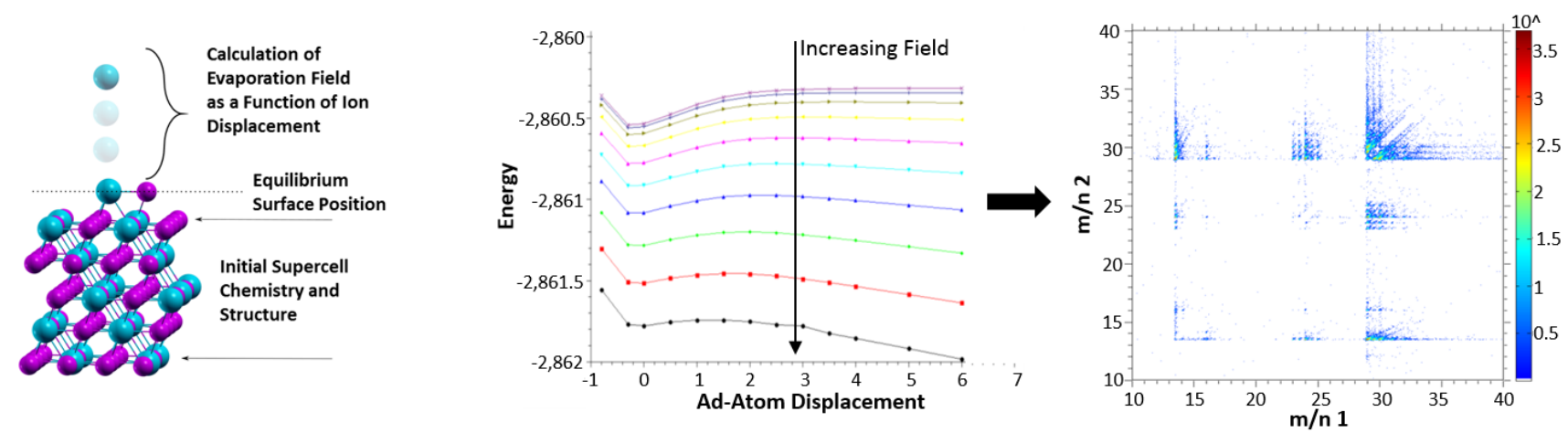

Figure 2. First principles procedure for calculating evaporation field of an atom as a function of neighborhood chemistry and structure and linking to evaporation physics, such as for the example ion evaporation map shown. The calculation operates by measuring the field change as the ion is pulled away from the surface. These calculations are to be performed for $\mathrm{N}$ in the different material layers as described. The measure in change of energy versus displacement for the different applied fields defines the evaporation field for the atom. This then allows us to compare the pair-wise interactions of surface atoms. 